\title{
A phase IIa randomised clinical study of GNbAC1, a humanised monoclonal antibody against the envelope protein of multiple sclerosis- associated endogenous retrovirus in multiple sclerosis patients
}

\author{
Tobias Derfuss, François Curtin, Claudia Guebelin, Claire Bridel, Maria Rasenack, Alain Matthey, \\ Renaud Du Pasquier, Myriam Schluep, Jules Desmeules, Alois B Lang, Hervé Perron, Raphael \\ Faucard, Hervé Porchet, Hans-Peter Hartung, Ludwig Kappos and Patrice H Lalive
}

Correspondence to: François Curtin

GeNeuro SA, 18 Chemin des Aulx, CH-1228 Plan-les-Ouates/Geneva, Switzerland. fc@geneuro.com

Tobias Derfuss, Claudia Guebelin, Maria Rasenack, Ludwig Kappos Neurology Department, Basel University Hospital, Switzerland

François Curtin, Alois B Lang, Hervé Perron, Raphael Faucard GeNeuro SA, Switzerland

Claire Bridel Department of Clinical Neurosciences, Geneva University Hospital, Switzerland

Alain Matthey, Jules Desmeules Pharmacology and Toxicology Division, Geneva University Hospital, Switzerland

Renaud Du Pasquier Myriam Schluep Neurology Department, Lausanne University Hospital, Switzerland

Hervé Porchet GeNeuro SA, Switzerland/Pharmacology Department, University of Pretoria, South Africa

Hans-Peter Hartung Neurology Department, Heinrich Heine University, Germany

Patrice H Lalive Department of Clinical Neurosciences, Geneva University Hospital, Switzerland/Department

of Genetic and Laboratory Medicine, Geneva University Hospital, Switzerland/Department of Pathology and Immunology, University of Geneva, Switzerland

\section{Abstract}

Background: $\mathrm{GNbAC1}$ is an immunoglobulin (IgG4) humanised monoclonal antibody against multiple sclerosis-associated retrovirus (MSRV)-Env, a protein of endogenous retroviral origin, expressed in multiple sclerosis (MS) lesions, which is pro-inflammatory and inhibits oligodendrocyte precursor cell differentiation. Objective: This is a randomised, double-blind placebo-controlled dose-escalation study followed by a six-month open-label phase to test GNbAC1 in MS patients. The primary objective was to assess GNbAC1 safety in MS patients, and the other objectives were pharmacokinetic and pharmacodynamic assessments. Methods: Ten MS patients were randomised into two cohorts to receive a single intravenous infusion of $\mathrm{GNbAC1} / \mathrm{placebo}$ at doses of 2 or $6 \mathrm{mg} / \mathrm{kg}$. Then all patients received five infusions of GNbAC1 at 2 or $6 \mathrm{mg} / \mathrm{kg}$ at four-week intervals in an open-label setting. Safety, brain magnetic resonance imaging (MRI), pharmacokinetics, immunogenicity, cytokines and MSRV RNA expression were studied.

Results: All patients completed the study. GNbAC1 was well tolerated in all patients. GNbAC1 pharmacokinetics is dose-linear with mean elimination half-life of 27-37 d. Anti-GNbAC1 antibodies were not detected. Cytokine analysis did not indicate an adverse effect. MSRV-transcripts showed a decline after the start of treatment. Nine patients had stable brain lesions at MRI.

Conclusion: The safety, pharmacokinetic profile, and pharmacodynamic responses to GNbAC1 are favourable in MS patients over a six-month treatment period.

Keywords: Multiple sclerosis, endogenous retrovirus, human endogenous retrovirus type W family, multiple sclerosis-associated retrovirus, monoclonal antibody, clinical trial

\section{Introduction}

$\mathrm{GNbAC} 1$ is a recombinant humanised monoclonal antibody of the IgG4/kappa class. Contrary to many drugs registered for multiple sclerosis (MS) with an immunomodulating or immuno-suppressing mode of action, ${ }^{1}$ GNbAC1 selectively binds the extracellular domain of a protein called multiple sclerosis-associated retrovirus (MSRV)-Env expressed by genes from the human endogenous retrovirus type $\mathrm{W}$ family $(\mathrm{HERV}-\mathrm{W})$ also named 'multiple sclerosis-associated retrovirus' (MSRV). ${ }^{2-4}$ Numerous studies have demonstrated links between the expression of MSRV-Env and MS. MSRV expression in the cerebrospinal fluid correlates with clinical progression and prognosis of MS. ${ }^{5}$ Immunohistochemical analyses of post-mortem brain tissue from MS patients localises the MSRV-Env protein to MS plaques with a higher protein expression in active plaques compared to inactive plaques. ${ }^{6-8}$ 
In MS pathogenesis, dysregulation of both innate and adaptive immune system is considered as the main triggering and/or exacerbating factor. MSRV-Env activates toll-like receptor 4 (TLR4) and has a proinflammatory effect mediated through its interaction with TLR4 in peripheral blood mononuclear cell (PBMC) cultures. ${ }^{9}$ Another effect of MSRV-Env is the blockade of the oligodendrocyte differentiation necessary for the remyelination process, also mediated by an interaction with TLR4 on oligodendrocyte precursor cells (OPCs). ${ }^{10}$ Based on the ability of MSRV-Env to activate the innate immune system and given its direct toxicity on OPCs, MSRV-Env is a relevant therapeutic target for MS.

MSRV-Env antagonist monoclonal antibody GNbAC1 had been studied first in a Phase I trial in 33 healthy subjects, showing a good safety as well as a linear pharmacokinetics. ${ }^{11}$ The goals of this placebocontrolled dose escalation study with an open-label extension phase were to assess the safety profile, immunogenicity, pharmacokinetic parameters and pharmacodynamics of repeated administrations of $\mathrm{GNbAC} 1$ at doses of 2 and $6 \mathrm{mg} / \mathrm{kg}$ in MS patients.

\section{Patients and methods}

\section{Trial design}

A Phase II study was performed in a single-blind, placebo-controlled dose-escalating randomised design in MS patients in two centres. In each of the two dose cohorts (doses $2 \mathrm{mg} / \mathrm{kg}$ or $6 \mathrm{mg} / \mathrm{kg}$ ) of five patients, four patients received $\mathrm{GNbAC1}$ and one patient received placebo in a sequential manner (single dose phase). Placebo patients were introduced to allow comparison between active and placebo treatments in case of safety issue at the first drug administration in patients, paralleling the design of the Phase I study. ${ }^{11}$ Then all patients of each cohort received GNbAC1 in a repeated dose phase either at 2 or $6 \mathrm{mg} / \mathrm{kg}$ dose in an open-label setting to get more information on the safety after repeated drug administrations.

A sample size of 10 patients was considered as sufficient for this first assessment of safety in patients.

Drug administrations in the repeated dose phase were performed at four-week intervals by intravenous infusion over one hour.

The primary objective of this study was to assess the safety and tolerability of single ascending doses, as well as of repeated administrations of GNbAC1 in MS patients. The secondary objectives were to determine the pharmacokinetics characteristics following administration of single ascending doses and repeated administrations of $\mathrm{GNbAC1}$ in MS patients; to determine pharmacodynamic markers of MS disease activity in patients including measurement of MSRV-Env markers and magnetic resonance imaging (MRI); to assess the immunogenicity of $\mathrm{GNbAC} 1$. No hypothesis testing was planned for this study, which was mainly explorative.

Patients. Male or female patients between 18-65 years, with primary progressive MS (PPMS), secondary progressive MS (SPMS) or relapsing-remitting MS (RRMS) (revised MacDonald criteria) ${ }^{12}$ with Expanded Disability Status Scale (EDSS) $\leq 6.5$, without MS treatment, were eligible. Details on inclusion/ exclusion criteria are provided in the Supplementary Material.

Prior to the start of the study, the study protocol and informed consent form were approved by the ethics committees of the two centres and the Swiss Medicine Agency, Swissmedic. All patients enrolled in this study had signed the written informed consent form prior to study entry.

Randomisation. Ten patients were chronologically assigned to one of the two escalating dose cohorts (2 $\mathrm{mg} / \mathrm{kg}$ and $6 \mathrm{mg} / \mathrm{kg}$ of $\mathrm{GNbAC} 1$ ). In each cohort, subjects were randomised to receive a single dose of either $\mathrm{GNbAC} 1$ or placebo at the ratio 4:1 for the first drug administration.

Study drug. GNbAC1 is an IgG4 humanised monoclonal antibody that selectively binds the extracellular domain of MSRV-Env. The antibody was humanised via an in silico design based on the amino acid sequence of a murine antibody that binds to MSRV-Env. GNbAC1 consists of two heavy and two light chains with enhanced disulphide linkage in the core region.

Treatment and safety monitoring. Each patient received the first administration of $\mathrm{GNbAC} 1$ or placebo sequentially. There was an interval of at least seven days between the GNbAC1 $2 \mathrm{mg} / \mathrm{kg}$ cohort and the $\mathrm{GNbAC} 16 \mathrm{mg} / \mathrm{kg}$ cohort to allow a safety review. A second safety review took place before starting the repeated administration phase.

Clinical laboratory evaluations and procedures. Blood and urine samples for clinical laboratory evaluations were collected and a 12-lead electrocardiogram (ECG) was recorded at screening, baseline and at each drug administration. Adverse events (AEs) were recorded at each visit. 
Pharmacokinetic assessment. Blood samples for pharmacokinetic assessment were collected at the following time points: pre-dose, 1, 2, 5, 13 and $24 \mathrm{~h}$ after start of infusion, at 4, 815 and $29 \mathrm{~d}$ after first administration and then before each repeated administration. The analysis of GNbAC1 was based on a competitive electrochemiluminescence (ECL)-based immunoassay using an anti-idiotypic monoclonal antibody (Mab1E4F7H6) against GNbAC1 as capture antibody. ${ }^{11}$ The following pharmacokinetic parameters for GNbAC1 were determined according to a model-independent pharmacokinetic analysis: area under the serum concentration versus time curve extrapolated to infinity $\left(\mathrm{AUC}_{0-} \infty\right)$; area under the serum concentration versus time curve from time zero to the last data point $\mathrm{t}_{\text {last }}$ above the limit of quantification $\left(\mathrm{AUC}_{0-\text { tlast }}\right)$; the maximum observed serum concentration $\left(\mathrm{C}_{\max }\right)$; the time to the maximum observed serum concentration $\left(\mathrm{t}_{\max }\right)$; the elimination half-life $\left(\mathrm{t}_{1 / 2}\right)$; the mean residence time (MRT); the total body clearance (CL); and the volume of distribution based on the terminal phase $\left(\mathrm{V}_{\mathrm{z}}\right)$. The pharmacokinetic analysis was performed using NC PKP.sas using SAS Version 9.2 (SAS Institute, Cary, North Carolina, USA).

Immunogenicity. Blood samples were taken before each $\mathrm{GNbAC} 1$ administration and 12 weeks after the sixth administration. The screening for binding antibodies against $\mathrm{GNbAC} 1$ was performed by ECL using a bridging format. GNbAC1 was labelled with biotin and with Sulfo-Tag, respectively. Both labelled preparations bind to anti-GNbAC1 antibodies. ${ }^{11}$ This complex was immobilised on streptavidin coated ECL-specific microtitre plates and was detected by using the ECL technique via the Sulfo-Tag $(\mathrm{Ru})$ label. This approach is based on the bridging format and enables the detection of all isotypes. Affinity purified monoclonal anti-idiotypic GNbAC1 antibodies served as controls. Positive samples were quasi-quantified by repeated analysis in dilution.

MRI. Brain MRI was performed at screening, $28 \mathrm{~d}$ after the first drug administration, and $28 \mathrm{~d}$ after the sixth administration. The following sequences were acquired: high-resolution isotropic T1-MPRage (3D) with gadolinium (Gd) ( $0.1 \mathrm{mmol}$ per $\mathrm{kg}$ body weight) and without $\mathrm{Gd}, 3 \mathrm{D}$ fluid attenuated inversion recovery (FLAIR), axial proton density (PD) (T2 long, with 3 $\mathrm{mm}$ slice thickness and no gap). Results were analysed in each centre.

MSRV-RNA analysis. MSRV-Env and Pol RNA was quantified in PBMCs by polymerase chain reaction (PCR) before first, third, and sixth administration.
The PCR analysis technique was based on a revised approach of the Mameli et al. ${ }^{6}$ method.

Cytokine analysis. Blood samples for cytokine measurement (interleukin 6 (IL-6), tumor necrosis factor $(\mathrm{TNF} \alpha)$, interferon (INF $\gamma)$ ) in serum were taken at the following time points: For the first administration: pre-dose, 2 , and $24 \mathrm{~h}$ after start of infusion, at 8, 15 and $29 \mathrm{~d}$ after infusion. For the second administration: pre-dose, at 8 , and $15 \mathrm{~d}$ after infusion. For the following administrations, blood samples were taken before start of the GNbAC1 infusion. The analysis was based on the quantitative sandwich enzyme immunoassay technique according to manufacturer instructions (Quantakine, RandD Systems Inc, Minneapolis, Minnesota, USA). The plates were measured with microplate reader MRX (Dynex Technologies GmbH, Denkendorf, Germany).

Statistical methods. Sample size considerations for this study were based on the usual sample size for safety assessment in early clinical phase studies. Descriptive statistics were presented for the pharmacokinetic, safety, and pharmacodynamic data using SAS Version 9.2 or higher (SAS Institute, Cary, North Carolina, USA). As this analysis was not planned in the study protocol, MSRV markers were analysed post-hoc with repeated measure analysis of variance (ANOVA), followed by pairwise comparisons with the Newman-Keuls method using SigmaStat 3.5 (Systat inc., San Jose, California, USA).

\section{Results}

\section{Demographic and other baseline characteristics}

Twelve patients were screened and 10 patients were included in the two centres and randomly assigned in one of the two cohorts, cohort $1(n=5)$ : single dose of GNbAC1 $2 \mathrm{mg} / \mathrm{kg}$ or placebo and repeated doses of GNbAC1 $2 \mathrm{mg} / \mathrm{kg}$; cohort $2(n=5)$ : single dose of GNbAC1 $6 \mathrm{mg} / \mathrm{kg}$ or placebo and repeated doses of GNbAC1 $6 \mathrm{mg} / \mathrm{kg}$ (Figure 1).

Demographic characteristics are summarised in Table 1 .

The demographic and disease characteristics of the patients were heterogeneous. However, this heterogeneity is not unwanted because it allows the safety and pharmacokinetic/-dynamic assessments in a broader range of patients. All the included patients completed the single dose and the repeated dose phases. 


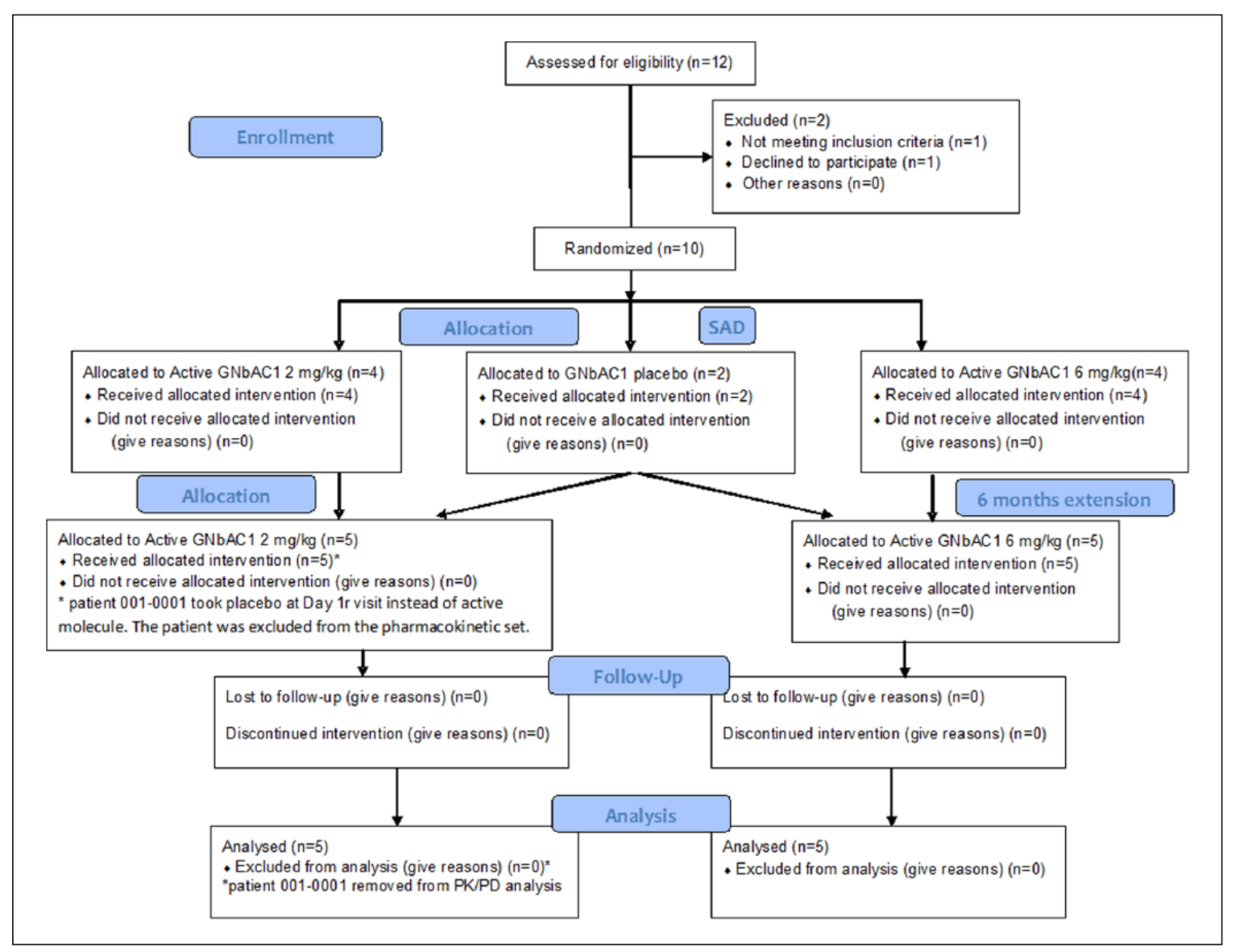

Figure 1. CONSORT 2010 flow diagram of participants through the study.

$\mathrm{PK} / \mathrm{PD}$ pharmacokinetics/pharmacodynamics SAD single ascending dose

Table 1. Demographic and disease characteristics of patients (mean \pm standard deviation).

\begin{tabular}{lllll}
\hline \multirow{2}{*}{ Demography and diagnosis } & GNbAC1 & & \\
\cline { 2 - 5 } & Placebo $(n=2)$ & $2 \mathrm{mg} / \mathrm{kg}(n=4)$ & $6 \mathrm{mg} / \mathrm{kg}(n=4)$ & Overall $(n=10)$ \\
\hline Age (years) & $50.5 \pm 7.8$ & $52.8 \pm 4.4$ & $55.5 \pm 8.9$ & $53.4 \pm 6.6$ \\
Female/male & $1 / 1$ & $1 / 3$ & $1 / 3$ & $3 / 7$ \\
Height $(\mathrm{cm})$ & $172.0 \pm 9.9$ & $177.8 \pm 13.8$ & $174.8 \pm 3.9$ & $175.4 \pm 9.2$ \\
Weight $(\mathrm{kg})$ & $71.8 \pm 11.0$ & $84.7 \pm 16.7$ & $84.1 \pm 1.1$ & $81.8 \pm 11.6$ \\
BMI $\left(\mathrm{kg} / \mathrm{m}^{2}\right)$ & $24.2 \pm 0.9$ & $26.6 \pm 2.5$ & $27.6 \pm 1.3$ & $26.5 \pm 2.1$ \\
Diagnosis & & & & $1(10.0)$ \\
RRMS, $n(\%)$ & $1(50.0)$ & $0(0.0)$ & $2(50.0)$ & $3(30.0)$ \\
PPMS, $n(\%)$ & $0(0.0)$ & $1(25.0)$ & $2(50.0)$ & $6(60.0)$ \\
SPMS, $n(\%)$ & $1(50.0)$ & $3(75.0)$ & $4.8+1.8$ & $4.8+1.5$ \\
EDSS & $3.3+1.1$ & $5.6+0.8$ & $15.8 \pm 12.3$ & $13.0 \pm 8.9$ \\
No. of years since start of illness & $6.0 \pm 1.4$ & $13.8 \pm 6.6$ & $3(75.0)$ & $5(50.0)$ \\
Prior MS treatment, $n$ (\%) & $0(0.0)$ & $2(50.0)$ & & \\
\hline BMI: body mass index; EDSS: Expanded Disability Status Scale; MS: multiple sclerosis; PPMS: primary progressive MS; RRMS: \\
relapsing-remitting MS; SPMS: secondary progressive MS.
\end{tabular}


Table 2. Treatment emergent adverse events in single dose phase and in open label extension, observed in more than $10 \%$ of patients, by system organ class (SOC) and preferred term (PT).

\begin{tabular}{|c|c|c|c|c|c|c|c|c|c|c|}
\hline \multirow[t]{3}{*}{ Single dose phase } & \multirow[t]{3}{*}{ SOC } & \multirow[t]{3}{*}{ PT } & \multicolumn{8}{|c|}{ GnbAC1 } \\
\hline & & & \multicolumn{2}{|c|}{$\begin{array}{l}\text { Placebo } \\
(n=2)\end{array}$} & \multicolumn{2}{|c|}{$2 \mathrm{mg} / \mathrm{kg}(n=4)$} & \multicolumn{2}{|c|}{$6 \mathrm{mg} / \mathrm{kg}(n=4)$} & \multicolumn{2}{|c|}{ Overall $(n=10)$} \\
\hline & & & $n$ & $\%$ & $n$ & $\%$ & $n$ & $\%$ & $n$ & $\%$ \\
\hline AEs & & & 2 & 100 & 3 & 75 & 4 & 100 & 9 & 100 \\
\hline $\begin{array}{l}\text { AE leading to } \\
\text { discontinuation }\end{array}$ & & & 0 & 0 & 0 & 0 & 0 & 0 & 0 & 0 \\
\hline \multirow[t]{5}{*}{ SAEs } & & & 0 & 0 & 0 & 0 & 0 & 0 & 0 & 0 \\
\hline & $\begin{array}{l}\text { General dis. and } \\
\text { admin. site cond. }\end{array}$ & Fatigue & 0 & 0 & 0 & 0 & 2 & 50 & 2 & 20 \\
\hline & $\begin{array}{l}\text { Infections and } \\
\text { infestations }\end{array}$ & Rhinitis & 0 & 0 & 1 & 25 & 1 & 25 & 2 & 20 \\
\hline & $\begin{array}{l}\text { Nervous system } \\
\text { disorders }\end{array}$ & Headache & 1 & 50 & 1 & 25 & 0 & 0 & 2 & 20 \\
\hline & & & & & \multicolumn{2}{|c|}{ GNbAC1 } & & & & \\
\hline \multirow{2}{*}{$\begin{array}{l}\text { Repeated dose } \\
\text { phase }\end{array}$} & & & & & \multicolumn{2}{|c|}{$2 \mathrm{mg} / \mathrm{kg}(n=5)$} & \multicolumn{2}{|c|}{$6 \mathrm{mg} / \mathrm{kg}(n=5)$} & \multicolumn{2}{|c|}{ Overall $(n=10)$} \\
\hline & & & & & $n$ & $\%$ & $n$ & $\%$ & $n$ & $\%$ \\
\hline AEs & & & & & 5 & 100 & 4 & 80 & 9 & 90 \\
\hline $\begin{array}{l}\text { AE leading to } \\
\text { discontinuation }\end{array}$ & & & & & 0 & 0 & 0 & 0 & 0 & 0 \\
\hline \multirow[t]{11}{*}{ SAEs } & & & & & 0 & 0 & 1 & 20 & 1 & 10 \\
\hline & Cardiac disorders & Sinus bradycardia & & & 1 & 20 & 1 & 20 & 2 & 20 \\
\hline & $\begin{array}{l}\text { General dis. and } \\
\text { admin. site cond. }\end{array}$ & Chest pain & & & 2 & 40 & 0 & 0 & 2 & 20 \\
\hline & & Fatigue & & & 1 & 20 & 1 & 20 & 2 & 20 \\
\hline & & Gait disturbance & & & 2 & 40 & 1 & 20 & 3 & 30 \\
\hline & $\begin{array}{l}\text { Infections and } \\
\text { infestations }\end{array}$ & Cystitis & & & 2 & 40 & 1 & 20 & 3 & 30 \\
\hline & & Nasopharyngitis & & & 2 & 40 & 1 & 20 & 3 & 30 \\
\hline & Investigations & $\begin{array}{l}\text { ECG QT } \\
\text { prolonged }\end{array}$ & & & 1 & 20 & 1 & 20 & 2 & 20 \\
\hline & & gGT increased & & & 2 & 40 & 1 & 20 & 3 & 30 \\
\hline & $\begin{array}{l}\text { Metabolism and } \\
\text { nutrition disorders }\end{array}$ & Hyperglycaemia & & & 0 & 0 & 2 & 40 & 2 & 20 \\
\hline & $\begin{array}{l}\text { Renal and urinary } \\
\text { disorders }\end{array}$ & Leukocyturia & & & 1 & 20 & 2 & 40 & 3 & 30 \\
\hline
\end{tabular}

\section{Safety evaluation}

Overall, 95 AEs occurred after the start of drug administration: 22 were experienced during the single dose phase; all AEs experienced during the single administration phase were rated as mild or moderate in intensity. In the repeated dose phase, 73 AEs were experienced. One AE was a serious adverse event (SAE): a biliary pancreatitis in a patient known for recurrent biliary lithiases was assessed as unlikely related to study treatment. Adverse events occurring in more than one patient are displayed in Table 2. There was no difference in the incidence of AEs between the two dose groups. There was also no particular pattern of AEs that could be attributed to the treatment.

\section{Pharmacokinetic evaluation}

Serum concentrations of GNbAC1 are summarised in Figure 2 with linear and semi-logarithmic scale presentations. The shape of the mean pharmacokinetic profiles suggests two exponential decline components. 


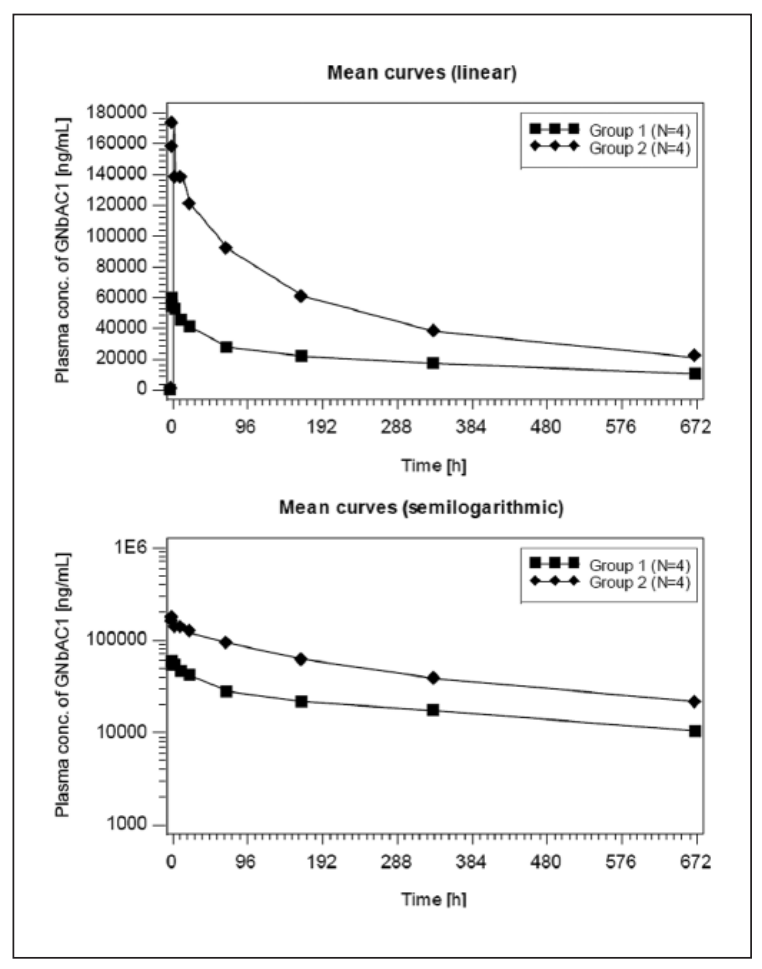

Figure 2. GNbAC1 blood concentration after first administration for group $1(2 \mathrm{mg} / \mathrm{kg})$ and group $2(6 \mathrm{mg} /$ $\mathrm{kg}$ ); linear and semi-logarithmic presentations.

The geometric mean pharmacokinetic parameter estimates (with coefficients of variation (CVs)) are reported in Table 3. The pharmacokinetics of GNbACl after intravenous administration in humans is characterised by a rather long apparent terminal elimination half-life of approximately $640-880$ hours $(27-37 \mathrm{~d})$. The $\mathrm{t}_{\max }$ values were observed at median times of $2 \mathrm{~h}$. Mean $\mathrm{C}_{\max }$ were about $59,000 \mathrm{ng} / \mathrm{ml}$ and $175,000 \mathrm{ng} / \mathrm{ml}$ for doses 2 and $6 \mathrm{mg} / \mathrm{kg}$ respectively. The ratios of AUC and $\mathrm{C}_{\max }$ between the 2 and $6 \mathrm{mg} / \mathrm{kg}$ doses are comprised between 2.1 and 3.0 supporting a linear pharmacokinetics of $\mathrm{GNbAC} 1$ within the evaluated dose range.

\section{Immunogenicity}

There was no evidence of antibody production against GNbAC1 throughout the entire study period. All the repeated measurements remained below the limit of detection.

\section{MRI}

Nine of 10 patients had stable MRI at six months compared to baseline; in the dose cohort $2 \mathrm{mg} / \mathrm{kg}$, one patient with SPMS presented a new T2 hyper-intense lesion in the cerebellum at the six-month brain MRI compared to baseline and day 28 MRIs.

\section{Cytokines}

No consistent changes were observed with cytokine measurements. Some patients presented sporadic slight increases of isolated cytokine values during the treatment period. However, due to the inconsistency of the cytokine concentrations at the different time points and because in none of the cases a consistent parallel or subsequent increase was observed there was no consistent correlation with $\mathrm{GNbAC} 1$ treatment.

\section{MSRV biomarkers}

MSRV-Env and MSRV-Pol transcripts were analysed: the distributions of MSRV transcripts levels at inclusion were homogeneous in both cohorts. Figure 3 presents measurements of MSRV-Env and MSRV-Pol in reference to GUS-B gene expression before first, third and sixth GNbAC1 administrations. There was a decrease in MSRV-Env and MSRV-Pol transcripts levels at three months and six months of treatment, the differences were statistically significant on the post-hoc analysis (repeated measure ANOVA on MSRV-Env $p=0.029$, on MSRV-Pol $p=0.044$ ).

\section{Discussion}

We report the first administration of GNbAC1 to MS patients. GNbAC1 is a monoclonal antibody targeting MSRV-Env, a protein which may play a critical role in MS by its inflammatory and myelinotoxic properties. The main objective of the study was to assess the safety profile of $\mathrm{GNbAC1}$ in MS patients, collecting pharmacokinetic data on repeated drug administration and explore responses to treatment on pharmacodynamic endpoints. The safety profile of repeated doses of GNbAC1 appears favourable in MS patients. Most of the adverse events were of mild to moderate severity. No dose-response relationship in frequencies or severity could be observed between the two dose cohorts. A serious adverse event, an acute biliary pancreatitis in a patient known for recurrent biliary lithiases, was reported but this event was not considered to be associated with the study drug. Most adverse events such as fluctuations in neurological symptoms or laboratory abnormalities observed during the treatment were either linked to direct or indirect fluctuations of MS disease or to preexisting medical conditions. Such events were not seen in healthy subjects receiving GNbAC1. ${ }^{11}$ Therefore, the reported AEs and laboratory abnormalities do not suggest a particular safety risk, allowing the conclusion that repeated intravenous administrations of $\mathrm{GNbACl} 1$ at 2 and $6 \mathrm{mg} / \mathrm{kg}$ are well tolerated and safe in MS patients.

No subject developed anti-drug antibodies during treatment, which points to a low immunogenicity of 
Table 3. Pharmacokinetic parameters by dose group, single GNbAC1 administration; values are geometric means (\% coefficient of variation).

\begin{tabular}{|c|c|c|c|}
\hline \multirow[t]{2}{*}{ Parameter } & \multicolumn{2}{|l|}{ GNbAC1 } & \multirow[t]{2}{*}{ Ratio } \\
\hline & $2 \mathrm{mg} / \mathrm{kg}$ & $6 \mathrm{mg} / \mathrm{kg}$ & \\
\hline $\mathrm{AUC}_{0 \text {-tlast }}(\mathrm{ng} \times \mathrm{h} / \mathrm{ml})$ & $18,601,334(21.0)$ & $46,108,575(24.4)$ & 2.5 \\
\hline $\mathrm{AUC}_{0 \text {-inf }}(\mathrm{ng} \times \mathrm{h} / \mathrm{ml})$ & $29,003,444(34.1)$ & $61,076,936(26.8)$ & 2.1 \\
\hline $\mathrm{C}_{\max }(\mathrm{ng} / \mathrm{ml})$ & $59,161(26.0)$ & $175,466(12.9)$ & 3.0 \\
\hline $\mathrm{T}_{\max }(\mathrm{h})$ & $5.3(102.0)$ & $5.5(94.5)$ & 1.0 \\
\hline$t_{1 / 2}(h)$ & $884(31.8)$ & $639(30.2)$ & 0.7 \\
\hline MRT (h) & 1197 (29.9) & $785(38.2)$ & 0.7 \\
\hline $\mathrm{CL}(\mathrm{ml} / \mathrm{kg} / \mathrm{h})$ & $0.076(35.5)$ & $0.104(27.6)$ & 1.4 \\
\hline $\mathrm{V}_{\mathrm{z}}(\mathrm{ml} / \mathrm{kg})$ & $88.6(8.3)$ & $92.5(28.0)$ & 1.0 \\
\hline \multicolumn{4}{|c|}{$\begin{array}{l}\mathrm{AUC}_{0 \text {-inf }} \text { : area under the serum concentration versus time curve extrapolated to infinity; } \mathrm{AUC}_{0 \text {-tlast }} \text { : area under the serum concentra- } \\
\text { tion versus time curve from time zero to the last data point } t_{\text {last }} \text { above the limit of quantification; } \mathrm{C}_{\text {max }}: \text { maximum observed serum } \\
\text { concentration; CL: total body clearance; MRT: mean residence time; } T_{\max }: \text { time to the maximum observed serum concentration; } t_{1 / 2} \text { : } \\
\text { elimination half-life in the terminal phase; } V_{z}: \text { volume of distribution based on the terminal phase. }\end{array}$} \\
\hline
\end{tabular}

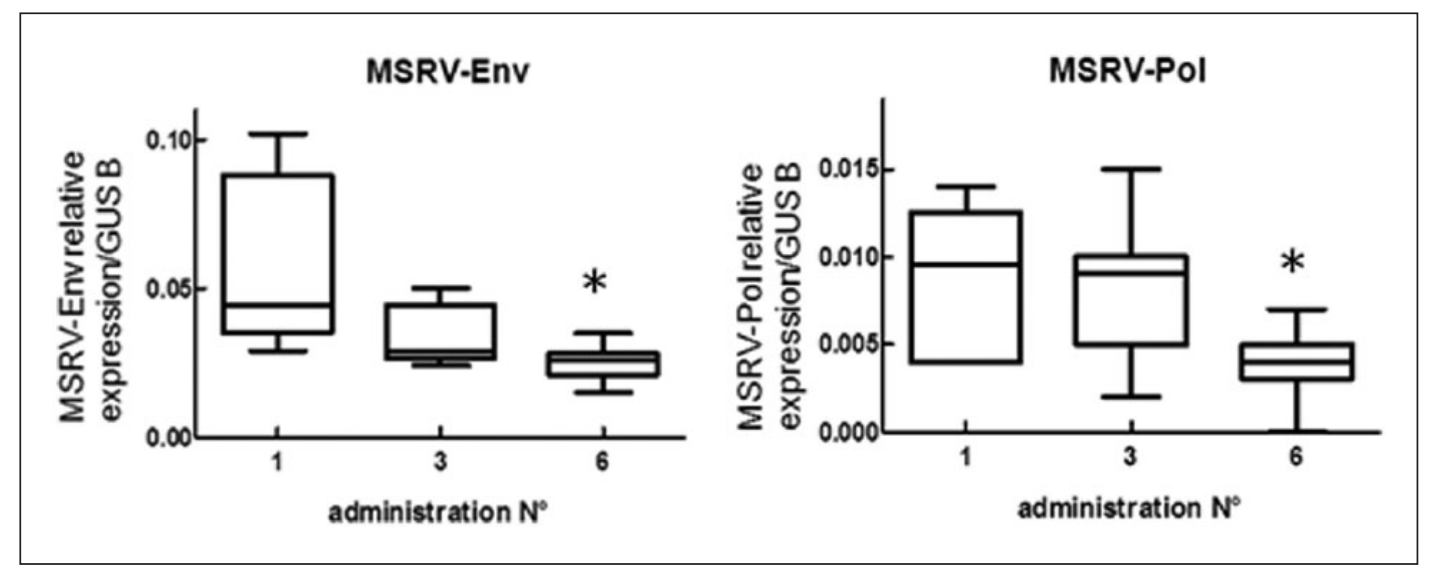

Figure 3. Multiple sclerosis-associated retrovirus (MSRV)-Env and MSRV-Pol transcripts expression proportional to reference beta-glucuronidase (GUS B), before first, third and sixth administrations (*pairwise comparison to baseline $p<0.05$, post-hoc repeated-measure analysis of variance).

$\mathrm{GNbAC1}$ and reduces the risk of neutralisation of GNbAC1. Nonetheless, the limited sample size and the relative short duration of follow-up limit somewhat these conclusions.

The observed pharmacokinetic profile of GNbAC1 suggests the presence of two exponential decline components. Geometric mean $t_{1 / 2}$ values ranged from 27 to $37 \mathrm{~d}$. These values are in line with the reported average $25 \mathrm{~d}$ for IgG in humans ${ }^{13}$ and with the values observed in healthy subjects at similar doses. ${ }^{11}$ The apparent shorter half-life of the $6 \mathrm{mg} /$ $\mathrm{kg}$ dose group compared to the $2 \mathrm{mg} / \mathrm{kg}$ dose group could possibly be explained by saturation phenomenon of IgG recycling by neonatal receptor of the cristallizable fragment $(\mathrm{FcRn})$ receptors as described with $\mathrm{IgG}$ at high dose, ${ }^{14}$ but considering the small number of subjects, these results should be interpreted with caution. The GNbAC1 half-life is compatible with a therapeutic administration schedule of one administration of GNbAC1 every four weeks.

Concentrations observed in this study are similar in the $2 \mathrm{mg} / \mathrm{kg}$ group and higher in the $6 \mathrm{mg} / \mathrm{kg}$ group compared to concentrations observed in healthy subjects and are therefore well above the minimal GNbAC1 concentrations of at least $4500 \mathrm{ng} / \mathrm{ml}$ needed to neutralise the MSRV-Env target protein. ${ }^{11}$ The exposure to GNbAC1 increases with the administered dose by a factor between two and three which is compatible with a linear increase of $\mathrm{GNbAC} 1$ exposure with dose. This dose-proportionality of $\mathrm{GNbAC1}$ pharmacokinetics 
also seen in healthy subjects ${ }^{11}$ is expected with humanised $\mathrm{IgG}$ monoclonal antibodies.

In terms of MRI assessment, nine out of 10 patients showed stable MRI images over the six-month treatment. There was no indication of a paradoxical increase of inflammation. The stability of the brain lesions over six months is an encouraging sign in terms of pharmacodynamic response to the treatment. However, due to the small sample size, the short observation period, and the inclusion of primarily progressive patients, we cannot draw firm conclusions about the efficacy of this treatment.

There was a decrease in the expression of the two MSRV biomarkers, MSRV-Env and Pol RNA transcripts, at three and six months of treatment, reaching statistical significance in a post-hoc repeated measures ANOVA. This response is interesting as it shows an effect not only on the protein itself but also on the endogenous retroviral transcriptional activity associated with MSRV-Env protein expression. This suggests that neutralising the MSRV-Env protein also down-regulates the expression of the corresponding endogenous retroviral genome. Moreover two reference MS treatments induce similar responses: interferon beta induces a decrease of MSRV-Env RNA measured by RT-PCR observed already at three months ${ }^{15}$ while natalizumab induces a decrease of MSRV-Env mRNA, measured by RT-PCR, observed later after six months of treatment. ${ }^{16}$ This pharmacodynamic response paralleling the response observed with MS treatments natalizumab and interferon beta is promising in terms of therapeutic efficacy. The exploration of these markers in a future proof of concept efficacy trial of GNbAC1 will allow to assess whether these MSRV markers could be a predictor of a therapeutic response to treatment.

Cytokine profiles based on TNF $\alpha$, IL-6 and IFN $\gamma$ showed no particular trends over the six months of analysis. As observed with interferon $\beta$ and natalizumab therapy, cytokine changes can only be expected over long term treatment, i.e. 1-3 years. ${ }^{17,18}$ and become significant only in larger patient populations. Thus the period of observation in this study is probably too short and the patient population too small to draw definitive conclusions on an influence of $\mathrm{GNbAC} 1$ on the cytokine profile.

In conclusion, repeated doses of $\mathrm{GNbAC} 1$ at doses 2 and $6 \mathrm{mg} / \mathrm{kg}$ were very well tolerated by MS patients over six consecutive administrations. No signs of induction of immunogenicity were identified.
GNbAC1 pharmacokinetics is dose-linear and its 27-37 $\mathrm{d}$ elimination half-life is compatible with a four-week administration schedule. MRI did not indicate a paradoxical increase of inflammation. The reduction of MSRV-Env RNA similar to that seen with interferon beta and natalizumab supports a pharmacodynamic response. GNbAC1, a monoclonal antibody antagonist of MSRV-Env, represents a totally new approach for the treatment of MS without a direct impact on the immune system. Based on the potential critical role of MSRVEnv in the MS pathophysiology, this therapeutic approach could be a novel and important addition to the armamentarium of MS treatments. The efficacy of this novel approach must now be shown in a proof of concept trial. The favourable safety results of this first study in MS patients provide a solid basis for these future studies.

\section{Acknowledgements}

The authors wish to thank Arno Kromminga from IPM Biotech for pharmacokinetic, cytokine and immunogenicity analyses.

\section{Conflict of interest}

Tobias Derfuss serves on scientific advisory boards for Novartis Pharma, Merck Serono, Biogen Idec, Genzyme, Teva, GeNeuro, Mitsubishi Pharma and Bayer Schering Pharma; has received funding for travel and/or speaker honoraria from Biogen Idec, Novartis Pharma, Merck Serono, Genzyme, and Bayer Schering Pharma and received research support from Novartis Pharma, Merck Serono, the Swiss National Science Foundation, the German Research Foundation, the European Union and the Swiss MS Society.

Renaud Du Pasquier has served on scientific advisory boards for Biogen Idec, Merck Serono, Teva, and Novartis; has received funding for travel or speaker honoraria from Abbvie, Biogen Idec, Teva, Merck Serono, and Bayer Schering Pharma.

Myriam Schluep has served as a consultant for MerckSerono, has received honoraria, payment for development of educational presentations and travel support from Merck-Serono, Biogen Dompé, Novartis, Sanofi-Aventis and Bayer Schering.

Hans-Peter Hartung received, with approval of the rector of Heinrich-Heine-University, fees for consulting and speaking from Bayer Healthcare, Biogen Idec, Genzyme, GeNeuro, Merck Serono, Novartis, Roche, Sanofi and Teva.

Ludwig Kappos participated as principal investigator, member or chair of steering committees or advisory boards in trials sponsored by Actelion, Addex, Allozyne, Bayer Health Care Pharmaceuticals, Bayer Schering Pharma, Biogen Idec, CLC Behring, GeNeuro 
SA, Genzyme, GlaxoSmithKline, Lilly, Merck Serono, Mitsubishi Pharma, Novartis, Octapharma, Ono Pharma, Praxicon, Roche, Sanofi-Aventis, Santhera, Siemens, Teva, and Xenoport. Honoraria and other payments for all these activities have been exclusively used for funding of research of his department.

Patrice H Lalive received honoraria for speaking from Biogen-Idec, Merck Serono, Novartis, Sanofi-Aventis, Teva; consulting fees from Biogen-Idec, GeNeuro, Genzyme, Merck Serono, Novartis, Sanofi-Aventis, Teva; research grants from Biogen-Idec, Merck Serono, Novartis.

François Curtin, Alois B Lang, Hervé Perron are all employees and shareholders of GeNeuro SA.

Raphael Faucard and Hervé Porchet are employees of GeNeuro SA.

Claudia Guebelin, Claire Bridel, Maria Rasenack, Alain Matthey and Jules Desmeules report no disclosure.

\section{Funding}

Study funding: GeNeuro SA, Geneva, Switzerland (ClinicalTrials.gov Identifier: NCT01639300).

\section{References}

1. Curtin F and Hartung HP. Novel therapeutic options for multiple sclerosis. Expert Rev Clin Pharmacol 2014; 7: 91-104

2. Perron H, Geny C, Laurent A, et al. Leptomeningeal cell line from multiple sclerosis with reverse transcriptase activity and viral particles. Res Virol 1989; 140: 551-561.

3. Perron H, Lalande B, Gratacap B, et al. Isolation of retrovirus from patients with multiple sclerosis. Lancet 1991; 337: 862-863.

4. Perron H, Garson JA, Bedin F, et al. Molecular identification of a novel retrovirus repeatedly isolated from patients with multiple sclerosis. The Collaborative Research Group on Multiple Sclerosis. Proc Natl Acad Sci U S A 1997; 94: 7583-7588.

5. Sotgiu S, Mameli G, Serra C, et al. Multiple sclerosisassociated retrovirus and progressive disability of multiple sclerosis. Mult Scler 2010; 16: 1248-1251.

6. Mameli G, Astone V, Arru G, et al. Brains and peripheral blood mononuclear cells of multiple sclerosis (MS) patients hyperexpress MS-associated retrovirus/HERV-W endogenous retrovirus, but not Human herpesvirus 6. J Gen Virol 2007; 88: 264-274.

7. Perron H, Germi R, Bernard C, et al. Human endogenous retrovirus type $\mathrm{W}$ envelope expression in blood and brain cells provides new insights into multiple sclerosis disease. Mult Scler 2012; 18: 1721-1736.

8. Perron $\mathrm{H}$ and van Horssen J HERV-W Env protein is strongly upregulated in inflammatory multiple sclerosis lesions. Poster P355 presented at ECTRIMS, Copenhagen, 2-5 October 2013.

9. Rolland A, Jouvin-Marche E, Viret C, et al. The envelope protein of a human endogenous retrovirus-W family activates innate immunity through CD14/TLR4 and promotes Th1-like responses. J Immunol 2006; 176: 7636-7644.

10. Kremer D, Schichel T, Förster M, et al.. Human endogenous retrovirus type $\mathrm{W}$ envelope protein inhibits oligodendroglial precursor cell differentiation. Ann Neurol 2013; 74: 721-732.

11. Curtin F, Lang AB, Perron H, et al. GNbAC1, a humanized monoclonal antibody against the envelope protein of multiple sclerosis-associated endogenous retrovirus: A first-in-humans randomized clinical study. Clin Ther 2012; 34: 2268-2278

12. Polman $\mathrm{CH}$, Reingold $\mathrm{SC}$, Banwell B, et al. Diagnostic criteria for multiple sclerosis: 2010 Revisions to the McDonald criteria. Ann Neurol 2011; 69: 292-302.

13. Wang W, Wang EQ and Balthasar JP. Monoclonal antibody pharmacokinetics and pharmacodynamics. Clin Pharmacol Ther 2008; 84: 548-558.

14. Mould DR and Green B. Pharmacokinetics and pharmacodynamics of monoclonal antibodies: Concepts and lessons for drug development. BioDrugs 2010; 24: 23-39.

15. Mameli G, Serra C, Astone V, et al. Inhibition of multiple-sclerosis-associated retrovirus as biomarker of interferon therapy. J Neurovirol 2008; 14: 73-77.

16. Arru G, Leoni S, Pugliatti M, et al. Natalizumab inhibits the expression of human endogenous retroviruses of the $\mathrm{W}$ family in multiple sclerosis patients: A longitudinal cohort study. Mult Scler 2014; 20: 174-182.

17. Stępień A, Chalimoniuk M, Lubina-Dąbrowska N, et al. Effects of interferon $\beta$-1a and interferon $\beta-1 b$ monotherapies on selected serum cytokines and nitrite levels in patients with relapsing-remitting multiple sclerosis: A 3-year longitudinal study. Neuroimmunomodulation 2013; 20: 213-222

18. Ramos-Cejudo J, Oreja-Guevara C, Stark Aroeira $\mathrm{L}$, et al. Treatment with natalizumab in relapsingremitting multiple sclerosis patients induces changes in inflammatory mechanism. J Clin Immunol 2011; 31: 623-631. 